\title{
Plug \& Produce auf dem Sprung in den Markt
} Neuerungen in Spezifikation und Implementierung des MTP

Modulare Automatisierung wird als eine der Schlüsseltechnologien zur Erhöhung der Flexibilität von Produktionsanlagen angesehen. Zugleich kann durch eine durchgängige Modularisierung von Produktionsanlagen und die Verwendung von Standards der Engineeringaufwand erheblich reduziert werden. Um dieses Ziel zu erreichen, arbeiten Namur (Interessengemeinschaft Automatisierungstechnik der Prozessindustrie) und der ZVEI (Zentralverband Elektrotechnik- und Elektronikindustrie) seit 2015 gemeinsam an der Spezifikation einer funktionalen, herstellerunabhängigen Beschreibung von Prozessmodulfunktionen, die aus dem Automatisierungsengineering eines Moduls heraus gewonnen werden kann. Durch den Import eines Module Type Packages (MTP) in eine übergeordnete Automatisierungsebene können dort die Modulfunktionen automatisch bereitgestellt werden. Durch die Kooperation mit dem Fachausschuss 5.16 der VDI/VDE-Gesellschaft Mess- und Automatisierungstechnik (GMA) sind Teile der gemeinsamen Spezifikationen in die Richtlinienreihe VDI/VDE/Namur 2658 überführt worden. Diese bilden nun die Grundlage zur Entwicklung entsprechender Werkzeuge auf Seiten der Automatisierungshersteller, die bereits in Pilotprojekten erprobt wurden. Der Artikel stellt den Fortschritt bei der Spezifikation und Standardisierung des MTP dar und zeigt Ergebnisse aus Pilotprojekten, die den Nutzen des MTP und die damit einhergehende praxistaugliche Realisierung von Plug \& Produce-Konzepten nachweisen.

SCHLAGWÖRTER Modulare Prozessautomation / Standardisierung /

MTP-basiertes Engineering

Plug \& produce nears market readiness -

Progress in the specification and implementation of MTPs

Modular automation is a key technology for increasing the flexibility of production facilities. At the same time, the continuous modularization of production facilities and the use of standards can significantly reduce the engineering requirements. To achieve this goal, Namur and ZVEI have been working together since 2015 on the specification of a functional, manufacturer-independent description of process module functions, which can be obtained from the modules automation engineering. By importing this module type package into a higher automation level, all functions can be provided there automatically. Due to the cooperation with the GMA 5.16, parts of the common specifications have been transferred to VDI/VDE/Namur 2658 series of standards. These now provide the basis for the development of appropriate tools by the automation manufacturers. The results from pilot projects are presented. 


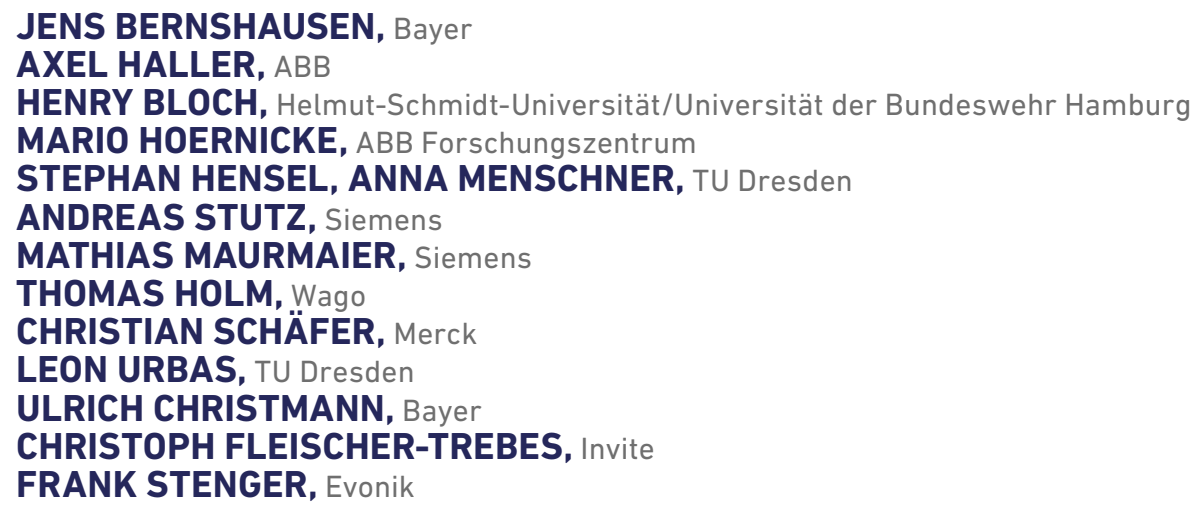

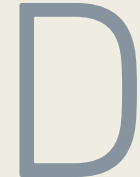

ie vierte industrielle Revolution und die hiermit einhergehenden Bestrebungen zur Digitalisierung führen zu einer Änderung des Marktes beziehungsweise Marktverhaltens. Durch die Spezialisierung von Produkten auf kleinere Marktgruppen bei gleichzeitiger Verkürzung der Produktlebenszyklen ist die Flexibilisierung des Produktionsprozesses von großer Bedeutung. Dies gilt insbesondere für die Prozessindustrie.

Prozesse müssen zuverlässig, reproduzierbar, fehlerfrei und im laufenden Betrieb anpassbar sein. Die Produktionskapazität soll kurzfristig erhöht oder auch vermindert werden können, um der Volatilität des Marktes gerecht zu werden.

\section{EINLEITUNG}

\subsection{Motivation}

Der Markt verändert sich schneller als je zuvor und die Hersteller sind gezwungen, ihre Produktion flexibel sowie konfigurierbar zu gestalten und gleichzeitig die Produktionseffizienz und -kosten niedrig zu halten. Neue digitale Möglichkeiten diversifizieren die Produktpalette stark, da die einzelnen Produkte kundenorientierter werden. Kürzere Produktlebenszyklen und kleinere Produktvolumina sind die Folge dieses Übergangs. Auch werden neuartige Konzepte für das Engineering und den Betrieb chemischer Anlagen den Anlagenlebenszyklus auf eine bisher nicht denkbare Weise verändern. Darüber hinaus sieht sich die europäische Industrie insgesamt mit Wettbewerbern, insbesondere aus Asien, konfrontiert, die technologisch unvermindert aufholen und günstiger produzieren können. Für die europäische Industrie ist es daher zwingend notwendig, den technologischen Fortschritt durch die Entwicklung neuer Ideen und Konzepte voranzutreiben, um wettbewerbsfähig zu bleiben.

Die Prozesstechnologie steht somit vor der Herausforderung, folgende Anforderungen erfüllen zu müssen, um wettbewerbsfähig zu bleiben:

- Time-to-Market verkürzen: Durch die Verkürzung der Produktlebenszyklen ist die notwendige Zeit zur
Markteinführung ein entscheidender Faktor bei der Wirtschaftlichkeitsbetrachtung eines Produktes.

- Erhöhte Flexibilität: Immer kleinere Produktmengen erhöhen den Bedarf an Anlagen, die mehr als ein Produkt produzieren können. Während dies in Batch-Prozessen bereits weit verbreitet ist, ist die Verwendung von Mehrzweck-Produktionsanlagen im kontinuierlichen Betrieb in der Regel immer noch eine große Herausforderung.

- Erhöhte Effizienz: Effizienzsteigerungen können durch die Wiederverwendung von Ingenieurleistungen aber auch durch flexibel nutzbares Prozessequipment erreicht werden. Dies kann zu erheblicher Reduktion des Engineering-Aufwands und der Kosten [1] sowie zu einer Vereinfachung der Neuordnung der Anlagen führen. Durch die Kombination dieser Effekte kann die Kosteneffizienz eines Prozesses enorm gesteigert werden.

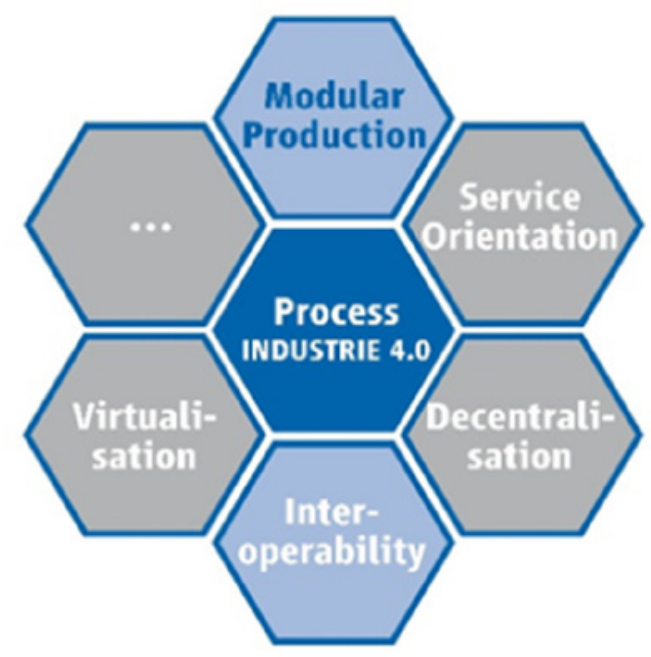

BILD 1: Industrie 4.0 in der Prozessindustrie 


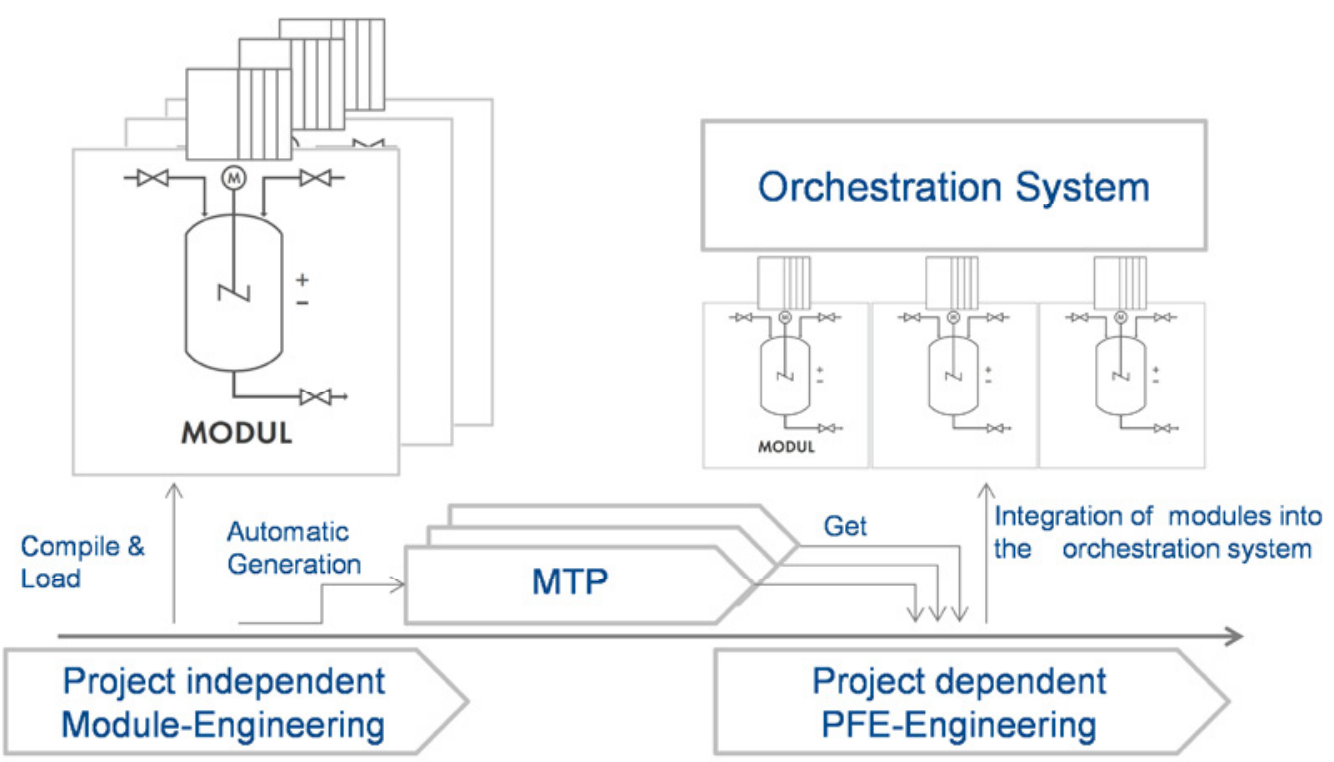

BILD 2: Engineering-Workflow für modulare Prozessanlagen [2]

Bild 1 zeigt einige wichtige Perspektiven, die die fortschreitende Digitalisierung für die Prozessindustrie eröffnet. Die Anwendung der Modularisierung wird unter anderem eine Schlüsselperspektive für eine chemische Industrie sein, die sich den oben genannten Herausforderungen stellt.

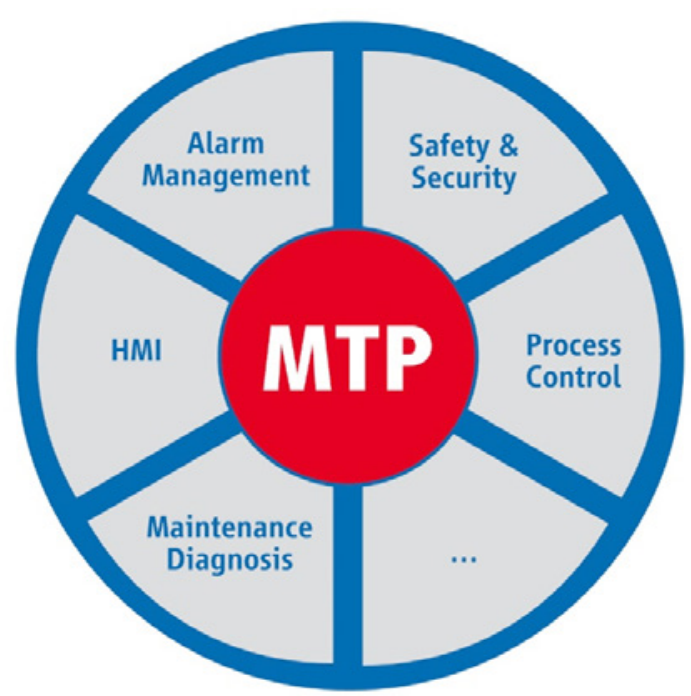

BILD 3: Grafische Darstellung der MTP-Inhalte [4]

\subsection{Modularisierung der Automation gemäß der NE 148}

Im Jahr 2015 initiierten Namur und ZVEI eine gemeinsame Initiative mit dem Ziel die Automatisierungssysteme hinsichtlich der Belange zur Flexibilisierung von Produktionsanlagen zu optimieren, also die Automatisierungssysteme zu modularisieren.

Im Fokus steht dabei der in Bild 2 dargestellte Engineering-Workflow.

Zentraler Baustein des Workflows ist die Generierung einer funktionalen, herstellerunabhängigen Beschreibung sowie die Kapselung der Modulfunktionalitäten [3]. Diese kann beispielsweise im Engineering des Leitsystems verwendet werden und fungiert als Schnittstellenbaustein, um die Funktionen des Prozessmoduls bekanntzumachen und anzusteuern. Das Konzept der modularen Automation wurde auf der Namur-Hauptsitzung 2015 präsentiert und in [4] beschrieben.

Diese in Bild 3 schematisch dargestellte Beschreibung des Module Type Package (MTP), wird in gemeinsamen Arbeitsgruppen von ZVEI und Namur spezifiziert. Innerhalb des MTP werden die Automatisierungsschnittstellen angegeben, die für die Kommunikation zwischen dem Automationssystem einer modularen Prozesseinheit (Process Equiment Assembly, PEA) und dem aufliegenden Orchestrierungssystem erforderlich sind. Im weiteren Verlauf werden die modularen Prozesseinheiten kurz als Modul oder Prozessmodul bezeichnet.

Das MTP dient zur Identifikation der Funktionalität und der Kommunikationsschnittstellen eines 


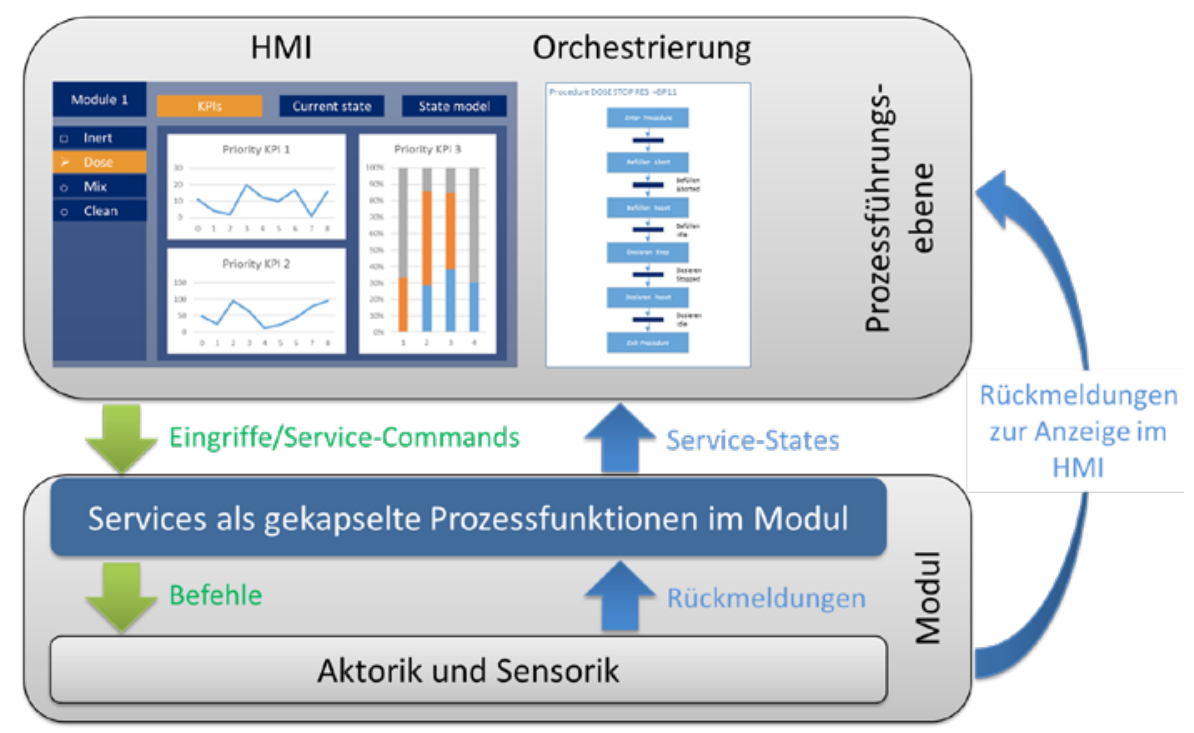

BILD 4: Grundlegende Kommunikation zur dienstbasierten Prozessführung [5]

Modulautomationssystems. Ferner beinhaltet es die Beschreibung des Bedienbildes, der Prozessfunktionen (Dienste), die das Modul bereitstellt, Information zu Diagnose und Maintenance sowie weitere für die Einbindung des Moduls in das Gesamtsystem wichtige Bestandteile. Es ist somit der Schlüssel für eine erhebliche Verringerung des Integrationsaufwands von Prozessmodulen und Package Units in die übergeordnete Prozessautomatisierung.

Teile dieser Spezifikation sind mittlerweile in die Richtlinie VDI/VDE/Namur 2658 [7-10] überführt worden.

\section{NEUERUNGEN IN DER SPEZIFIKATION DES MTP}

\subsection{Dienste und deren Orchestrierung}

Innerhalb des MTP werden verfahrenstechnische Funktionen in Diensten gekapselt und der Prozessführungsebene (Process Orchestration Layer, POL) zur Orchestrierung bereitgestellt. Im Folgenden wird der aktuelle Stand der Richtlinienarbeit des GMA-Fachausschusses 5.16 bezüglich des vierten Richtlinienblatts (VDI/VDE/Namur 2658) Dienstmodellierung dargestellt. Dieser Status entstand im Rahmen der Taskforce Prozessführung, bestehend aus Mitgliedern von ZVEI, Namur und GMA und wird aktuell noch weiter entwickelt. Die bislang getroffenen Definitionen werden in diesem Beitrag auszugsweise dargestellt.

\subsubsection{Dienste von Prozessmodulen}

modulare Prozessanlagen unterscheiden sich hinsichtlich der Kommunikationsarchitektur von herkömmlichen
Anlagen. Zentrales Element ist die Kapselung verfahrenstechnischer Funktionen in Diensten innerhalb der Module. Diese Dienste werden in der Prozessführungsebene so orchestriert (zeitlich und logisch geeignet an- und abgefahren), dass der gewünschte Produktionsablauf in der jeweiligen modularen Anlage realisiert wird. Die Kommunikationsarchitektur ist in Bild $4 \mathrm{zu}$ sehen.

Im Gegensatz zu herkömmlichen Anlagen ist eine Einflussnahme auf einzelne Aktoren im Automatikbetrieb der Anlage nicht mehr möglich und notwendig. Aus dem POL werden Befehle an die Dienste der verschiedenen Module geschickt. Diese Dienste folgen dem Zustandsautomaten nach Bild 5, wobei innerhalb eines jeden Zustands Programme, zum Beispiel nach IEC 61131-3 [6], aufgerufen werden.

Innerhalb dieser Programme werden Befehle an die Aktoren gesendet und deren Rückmeldungen in gewohnter Weise verarbeitet. Aus dem Ablauf der Programme ergibt sich, wann ein Dienst abgeschlossen ist. Die Programme werden vom Dienst innerhalb des Moduls gesteuert und es wird dem Modulbauer überlassen, wann ein Dienst abgeschlossen ist. Basierend auf der Rückmeldung des Zustands, in dem sich der Dienst befindet, kann der POL durch weitere diensteorientierte Kommandos geeignet Einfluss nehmen (vergleiche Bild 4). Neben den Zuständen werden auch Prozesswerte im Bedienbild auf der Ebene des POL angezeigt (vergleiche [8]). Hierbei ist zu beachten, dass der Bediener im Automatikbetrieb der Dienste ausschließlich lesenden Zugriff auf die Aktoren der Feldebene besitzt und damit keinen 


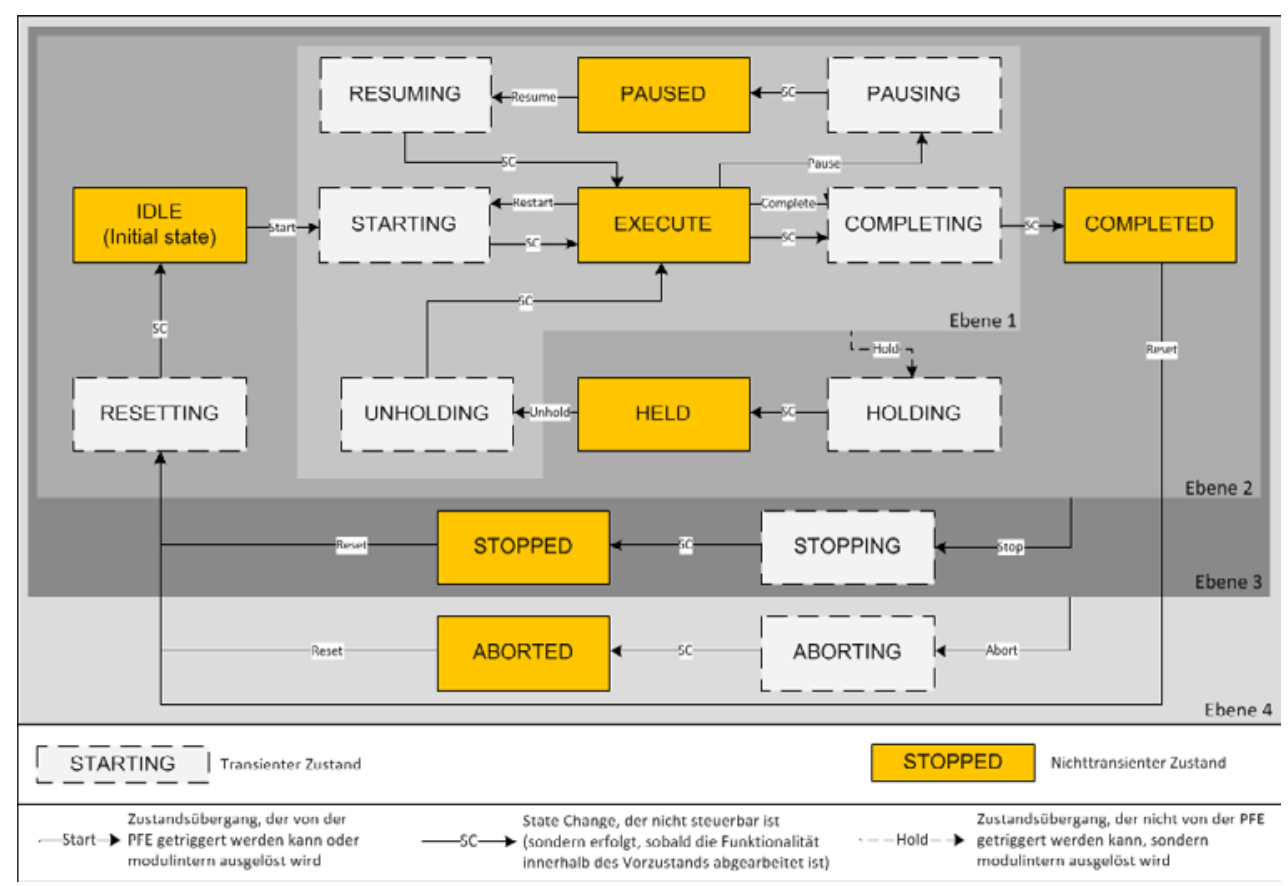

BILD 5: Zustandsautomat für Dienste [10]

direkten Steuerbefehl an einzelne Geräte senden kann.

Die Dienste der Module sind demnach als neue hierarchische Ebene in der Kommunikationsarchitektur zu verstehen, welche die Modulfunktionalitäten abstrahieren. Die Prozessführungsebene sagt dem Modul also nicht mehr, wie es etwas zu tun hat, sondern was es tun soll.

Die Beschreibung der Dienste wird Inhalt des vierten Richtlinienblatts der VDI/VDE/Namur Richtlinie 2658 [7-10] sein. Bisher sind hierfür folgende Aspekte initial festgelegt:

- Zustandsmodell für Dienste, inklusive der semantischen Definition der Zustände,

- Definition und Enummerierung der Steuer- und Statuswörter eines Dienstes,

- Definition und Modellierung von Fahrweisen,

- Definition der Betriebsarten eines Dienstes,

- Definition und Modellierung von Dienstparametern.

\subsubsection{Zustandsmodell für Dienste}

Der Zustandsautomat eines jeden Dienstes besteht aus 16 Zuständen in vier hierarchischen Ebenen. Die Struktur des Zustandsautomaten ist in Bild $5 \mathrm{zu}$ sehen. Diese Zustände werden durch einen Befehl (Anforderung eines Zustandsübergangs) erreicht und wechseln selbstständig nach Beendigung der in ihnen hinterlegten Funktion in den Folgezustand. Von diesem Ablauf wird abgewichen, sollte ein Befehl einer höheren Hierarchieebene des Automaten erfolgen. Der Zustandsautomat ist in vier Hierarchieebenen eingeteilt. In Ebene 1 liegen die Zustände Starting, Running, Completing, Pausing, Paused und Resuming. In Ebene 2 liegen die Zustände Idle, Completed, Resetting, Holding, Held und Unholding sowie Ebene 1. In Ebene 3 befinden sich die Zustände Stopping und Stopped sowie die Ebene 2. Der Ebene 4 sind die Zustände Aborting und Aborted sowie die Ebene 3 zugehörig. Die Hierarchieebenen beschreiben, dass aus einer niedrigeren Ebene zu jedem Zeitpunkt der Wechsel in eine höhere Ebene erfolgen kann. Der Wechsel in Ebene 2 erfolgt hierbei über den Befehl Hold, der Übergang in Ebene 3 erfolgt über den Befehl Stop, der Übergang in Ebene 4 erfolgt über den Befehl Abort.

Die Befehle sind an den Zustandsübergangs-Pfeilen dargestellt. Befehle in Rot sind nicht von dem POL anforderbar, sondern werden modulintern aufgelöst. Der Zustandsübergang SC steht für State Change und zeigt an, dass dieser Übergang durch den vorgelagerten Zustand ausgelöst wird. Diese Art des Zustandsüberganges ist ausschließlich nach transienten, also nicht stationären Zuständen vorgesehen. Der Zustandsautomat besteht aus transienten und nicht transienten Zuständen. Um transiente Zustände handelt es sich bei allen auf ,-ing“ endenden Zuständen mit der Ausnahme von Running [5].

Die durch den POL anforderbaren Zustandsübergänge sind: Start, Complete, Reset, Pause, Resume, 
Unhold, Stop, Abort und Restart. Das Modul darf intern jederzeit Zustandsübergänge selbst auslösen, dem POL wird lediglich der neue Zustand signalisiert. Das Zustandsmodell ist nicht konfigurierbar, das heißt, jeder Dienst muss dieses vollständig implementieren (Zustände und Zustandsübergänge). Zustände können hierbei eventuell keine Aktion im Modul erzeugen, müssen allerdings kurzzeitig beim Durchlaufen eine Rückmeldung an den POL geben und damit einmalig angezeigt werden. In mehreren Zuständen darf die gleiche Funktionalität implementiert sein, zum Beispiel in Stopping und Aborting.

\subsubsection{Steuer- und Statuswörter}

Um einen Dienst aus dem POL zu orchestrieren, verwendet jeder Dienst ein Steuer- und ein Statuswort. Sowohl das Steuer- als auch das Statuswort folgen einer definierten Enumeration [10]. Hierbei werden die Zustände vom Dienst an den POL und die Befehle von dem POL an den Dienst übermittelt.

Das Modul darf aufgrund vorliegender Prozesswerte oder Verriegelungen auf Einzelsteuerebene Zustandsübergänge zeitweise verriegeln. Hierfür wird für jeden Zustandsübergang ein Bit einer Verriegelungsvariablen verwendet. Dieses Bit zeigt an, ob ein Zustandsübergang aktuell möglich ist. Die Codierung der Variable ist [10] zu entnehmen. Gibt der Wert der Variable an, dass ein Befehl nicht anforderbar ist, so kann dieser Befehl nicht durch den Bediener oder den POL ausgelöst werden. Zunächst muss der Befehl wieder freigegeben werden. Als Beispiel wird ein Dosiermodul mit den Diensten Dosieren und Reinigen betrachtet. Befindet sich der Dienst Dosieren im Zustand Running, so ist der Start-Befehl des Dienstes Reinigen zu diesem Zeitpunkt verriegelt.

\subsubsection{Fahrweisen}

Um die Dienste der Module möglichst effizient schneiden zu können, dürfen Fahrweisen, zum Beispiel für den Dienst Temperieren die Fahrweisen Temperatur über Rampe anfahren und Temperatur halten, genutzt werden. Dies ermöglicht zum einen eine Zuordnung von Prozesswerten, die zum Betrieb des Dienstes notwendig sind und zum anderen die Fahrweise des Dienstes selbst. Fahrweisen können zu jeder Zeit verändert werden. Der Dienst übernimmt die aktuelle Fahrweise und die Prozesswerte allerdings immer nur im Zustand Starting.

Des Weiteren erfolgt die Unterscheidung von Diensten in selbstbeendende und kontinuierliche Dienste. Diese Unterscheidung erfolgt auf Ebene der Fahrweisen. Somit kann eine jede Fahrweise eines Dienstes entweder selbstbeendend oder kontinuierlich sein. Im Beispiel des Dienstes Temperieren ist die Fahrweise Temperatur über Rampe anfahren selbstbeendend sobald die Temperatur erreicht wurde, und die Fahrweise Temperatur halten ist kontinuierlich. Hat ein Dienst nur eine Fahrweise, die selbstbeendend ist,

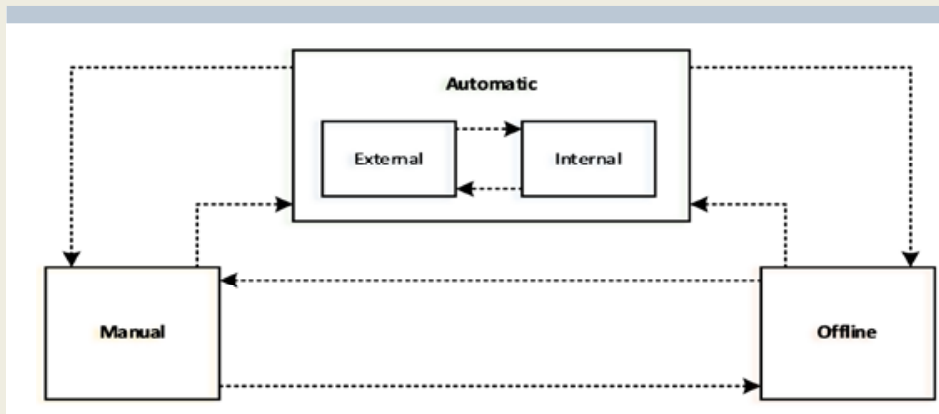

BILD 6: Betriebsarten eines Dienstes

ist der gesamte Dienst selbstbeendend. Ist diese Fahrweise kontinuierlich, so ist der Dienst kontinuierlich. Unterschiedliche Fahrweisen innerhalb eines Moduls sind erlaubt. Der POL muss Dienste mit verschiedenen Fahrweisen unterstützen. Innerhalb eines Moduls können Dienste so geschnitten sein, dass keine unterschiedlichen Fahrweisen für einen Dienst vorliegen. Eine vollständige Definition ist in [10] zu entnehmen

\subsubsection{Betriebsarten}

Die Betriebsart von Diensten wird wie die Betriebsart auf der Einzelsteuerebene [9] gesteuert. Hierzu wird die Schnittstelle ExtendedOperationMode, wie in [9] definiert, verwendet. Der Unterschied besteht darin, dass aus der Betriebsart Automatic auch direkt in die Betriebsart Offline, und umgekehrt, gewechselt werden darf (siehe Bild 6). Dies ist auf Einzelsteuerebene nicht möglich. Die vollständige Definition der Betriebsarten ist [4] zu entnehmen.

\subsubsection{Dienstparameter}

Die Ausführung eines Dienstes kann durch Parameter beeinflusst werden. Die Parameter können zum Beispiel Zeiten oder Sollwerte von Prozessparametern, wie Temperaturen und Drücke sein. Einzelne Sollwerte können in bestimmten Fahrweisen gültig sein, in anderen Fahrweisen nicht. Im MTP ist die Zuordnung, welche Fahrweise eines Dienstes welche Sollwerte nutzt, abgebildet. Zur Unterscheidung der Parameter wurden folgende Klassen definiert:

- Konfigurationsparameter werden verwendet, um grundlegende Einstellungen für einen Dienst vorzunehmen, bevor dieser ausgeführt wird. Die Konfigurationsparameter gehören immer zu Diensten, können in mehreren Diensten verwendet werden und werden immer dann vom Dienst übernommen, wenn dieser zur Ausführung vorbereitet wird oder aus der Betriebsart Offline in eine andere Betriebsart wechselt. Beispiele für Konfigurationsparameter sind zu nutzende Sensoren als Eingangswerte, Reglerparameter oder Grenzwerte vor- und nachgelagerter Module. 


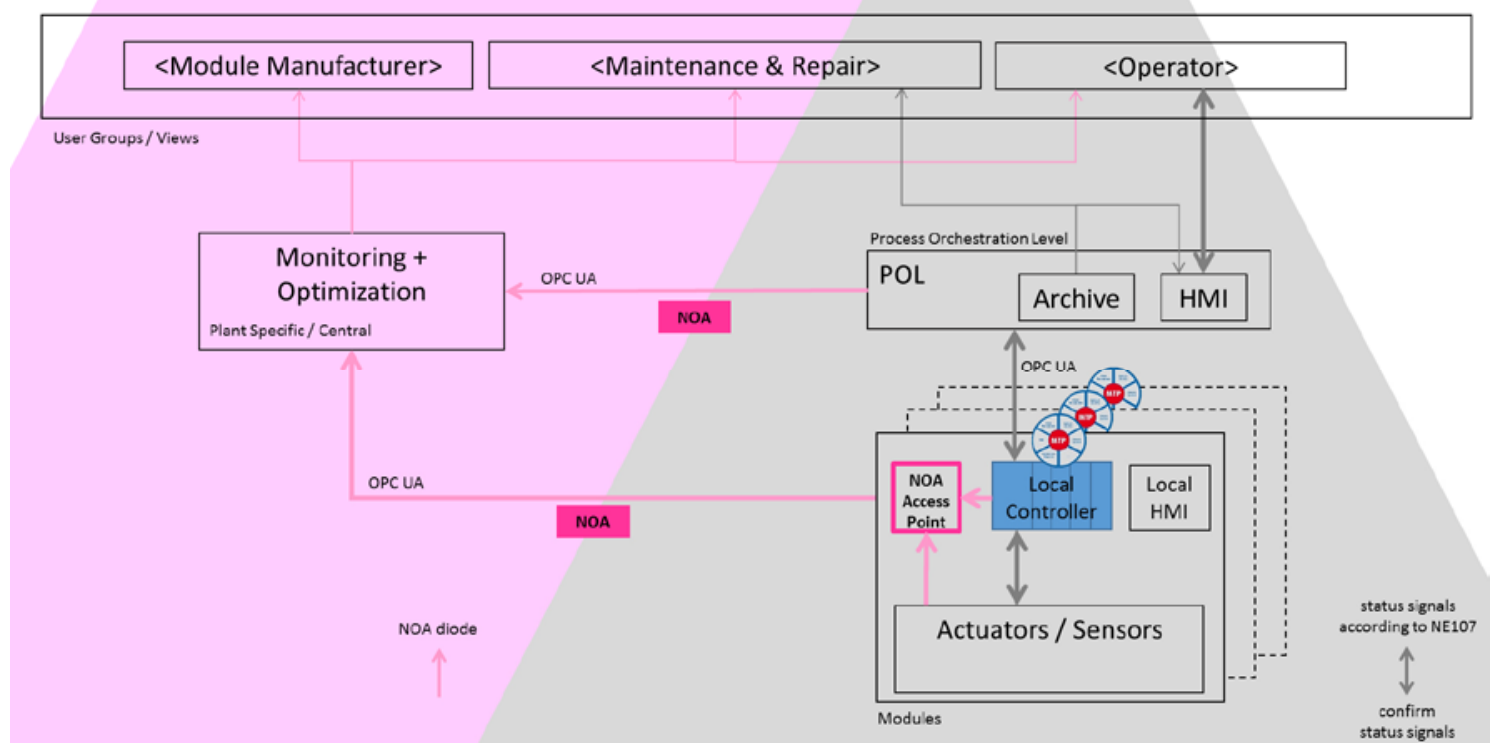

BILD 7: Diagnose und Maintenance in modularen Produktionsanlagen

- Fahrweisenparameter sind Fahrweisen zugeordnet. Sie werden eingestellt bevor ein Dienst gestartet oder neu gestartet wird. Immer wenn der Dienst in dem Zustand Starting wechselt, werden die Parameter übernommen und sind ab diesem Zeitpunkt gültig. Beispiele für Fahrweisenparameter sind orchestrierungsrelevante Sollwerte wie Temperaturvorgaben.

- Prozesswerte sind Parameter, die kontinuierlich gelesen oder geschrieben werden. Sie sind folglich

\begin{tabular}{|l|l|l|}
\hline Page & Description & Status \\
\hline $2658-1$ & General Concept and Interfaces & $\begin{array}{l}\text { White print DE/EN } \\
\text { expected Q4/2018 }\end{array}$ \\
\hline $2658-2$ & $\begin{array}{l}\text { Description of Operator } \\
\text { Interfaces to Process Equipment } \\
\text { Assemblies }\end{array}$ & $\begin{array}{l}\text { White print DE/EN } \\
\text { expected Q1/2019 }\end{array}$ \\
\hline $2658-3$ & $\begin{array}{l}\text { Interfaces and Libraries for Basic } \\
\text { Object Types }\end{array}$ & $\begin{array}{l}\text { Green print DE expected } \\
\text { Q4/2018 }\end{array}$ \\
\hline $2658-4$ & $\begin{array}{l}\text { Services for Process Equipment } \\
\text { Assemblies }\end{array}$ & $\begin{array}{l}\text { First proposal available } \\
\text { Green print expected } \\
\text { Q1/2019 }\end{array}$ \\
\hline $2658-5$ & $\begin{array}{l}\text { Run TimeAspects and } \\
\text { Communication }\end{array}$ & $\begin{array}{l}\text { Under specification } \\
\text { Green print expected } \\
\text { Q2/2019 }\end{array}$ \\
\hline 2658- & $\begin{array}{l}\text { Implementation Guideline } \\
\text { communication w/ OPC UA }\end{array}$ & $\begin{array}{l}\text { Green print expected } \\
\text { Q2/2019 }\end{array}$ \\
\hline 5.1 & & \\
\hline
\end{tabular}

BILD 8: Übersicht über den Standardisierungsfortschritt unabhängig von der Betriebsart oder dem aktuellen Zustand eines Dienstes. Prozesswerte können durch den POL gelesen oder bereitgestellt werden. Ferner können diese einem oder mehreren Diensten zugeordnet werden, jedoch nicht den Fahrweisen. Beispiele für Prozesswerte sind modulübergreifende Verriegelungen oder Reglerwerte für modulübergreifende Regelkreise.

- Reportwerte sind Werte, die als Nachweis und zur Dokumentation verwendet werden können. Ein Reportwert wird kontinuierlich aktualisiert. In den Zuständen Completed, Aborted und Stopped wird der Wert eingefroren. So kann dieser von einem angebundenen System gelesen werden.

Die vollständige Definition ist [10] zu entnehmen.

\subsection{Diagnose und Maintenance}

Die Kapselung von Modulfunktionaltitäten birgt im Hinbick auf die Diagnose- und Maintenance-Fähigkeit von Modulen im Lifecycle der Produktionsanlage einige Herausforderungen. Zum einen besteht die Möglichkeit, durch die interne Automatisierung die Diagnose- und Maintenanceinformationen für den Anlagenbetreiber situativ aufzubereiten und verdichtet zur Verfügung zu stellen. Zum anderen ist es im Hinblick auf Smart-Data-Ansätze und mögliche Prozessoptimierungen unerlässlich, den Zugang zu Detailinformationen bis in die Feldebene zu erhalten. Mit diesem Hintergrund wird aktuell in dem gemeinsamen Arbeitskreis Diagnose und Maintenance von 
Namur und ZVEI über einen zweiteiligen Ansatz diskutiert.

Dieser Ansatz ist in Bild 7 illustriert. Der dort grau dargestellte Bereich zeigt den Austausch von verdichteten Diagnose- und Maintenance-Informationen über die Kommunikationsstelle zwischen lokaler Modulsteuerung und Prozessorchestrierung. Diese Informationen gilt es im MTP zu modellieren. Der linke Bereich zeigt den Zugang zu Detailinformationen. Hier wird auf die Konzepte der Namur Open Architecture (NOA) vewiesen. Diese stellt einen zweiten Kommunikationskanal dar und dient dem nicht prozessrelevanten Austausch von Informationen parallel zu den Ebenen der Automatisierungspyramide.

Der Fokus der MTP-Spezifikation liegt folglich in der Definition der Kommunikation zwischen der Prozessmodul- und der Anlagenautomatisierungsebene. Grundsätzlich ist die Abstraktion der Diagnoseinformationen mittels der in der NE 107 [11] definierten Status denkbar. Neben den Sensor-/Aktorinformationen besteht zusätzlich die Notwendigkeit auch prozessrelevante Diagnoseinformationen, beispielsweise den Druckverlust über einen Filter, auf Modulebene zu erzeugen und über das MTP bekannt zu machen. Ob darüber hinaus weitere Informationen notwendig sind, ist Gegenstand aktueller Diskussionen innerhalb des Arbeitskreises Diagnose und Maintenance.

\section{STANDARDISIERUNGSAKTIVITÄTEN}

Für die Überführung der MTP-Spezifikation aus der Theorie in die Praxis ist die Erarbeitung einer Richtlinie wichtig. Dies schafft eine Basis, die es ermöglicht, Engineering-Werkzeuge zu entwickeln und $\mathrm{zu}$ kommerzialisieren, die einen herstellerneutralen MTP-Austausch ermöglichen.

Innerhalb des GMA-Fachausschusses 5.16 werden die Spezifikationen von ZVEI und Namur aufgearbeitet und in die Richtlinie VDI/VDE/Namur 2658 überführt. Analog zu der Aufteilung des MTPs in die unterschiedlichen Funktionen, ist auch diese Richtlinie in unterschiedliche Blätter aufgeteilt, die die verschiedenen Facetten beschreiben. Der Benefit der Aufteilung der Richtlinie besteht darin, dass die Standardisierung bereits funktioneller Teile des MTPs erfolgen kann, ohne die durchgängige Spezifikation des MTPs durchgeführt zu haben. Die standardisierten Elemente können dann in die Produkte der Automatisierungslieferanten überführt werden.

In Bild 8 ist der aktuelle Status der Richtlinie VDI/ VDE/Namur 2658 aufgeführt. Die ersten drei Richtlinienblätter sind bereits im Gründruck veröffentlicht. Blatt 1 beschreibt das generelle Konzept des MTPs sowie die Modellierung von statischen und dynamischen Daten über standardisierte Schnittstellen. Blatt 2 und 3 beschreiben den Import- und Exportmechanischmus für Bedienbilder und deren

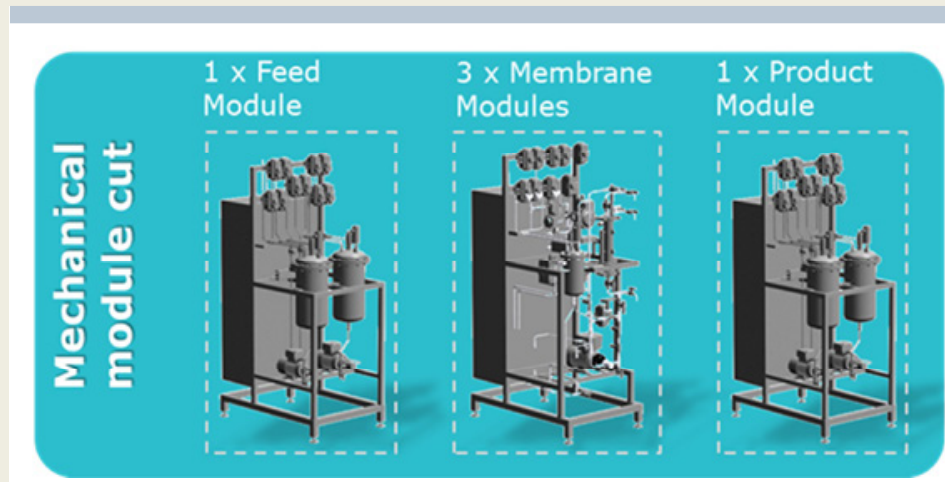

BILD 9: Modulschnitt der Membranfiltrationsanlage

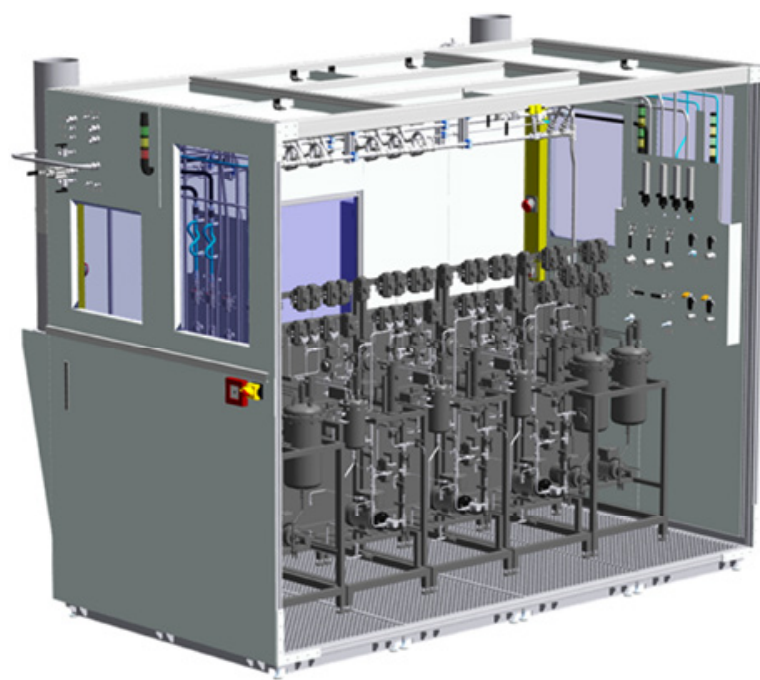

BILD 10: Infrastrukturmodul für die Prozessmodule

Modellierung innerhalb des MTPs. Weitere Blätter der Richtlinie werden in 2019 folg en.

\section{ERFAHRUNGEN AUS DER PILOTIERUNG}

Fortschritt innerhalb der Arbeiten zur Standardisierung kann nur durch eine prototypische Erprobung erfolgen, um hieraus wichtige Erkennisse für die weitere Abstimmung zu gewinnen. Neben den prototypischen Umsetzungen sind aber auch Erfahrungen aus der Praxis der Produktion wichtig, um neben offenen Entwicklungsfeldern auch Akzeptanz für diese neuen Entwicklungen zu schaffen. Erste Erfahrungen liegen hierzu vor, die im Folgenden beschrieben sind.

\subsection{Smart-Factory-Projekt von Merck}

Im Rahmen des Projekts Smart Factory will Merck einen weitreichenden Schritt in Richtung der modularen, kontinuierlichen Produktion von Fein- und 


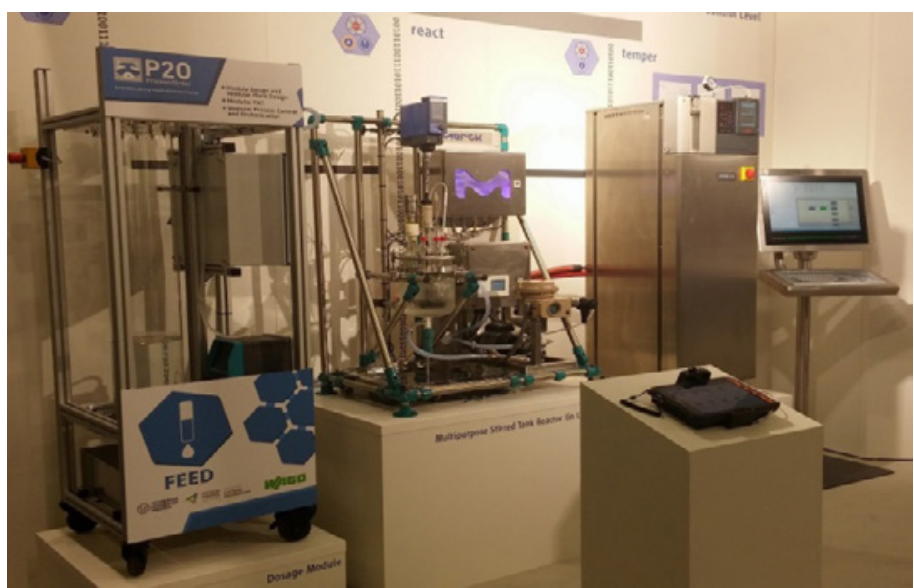

BILD 11: Funktionsfähiger Demonstrator auf der Achema 2018 [12]

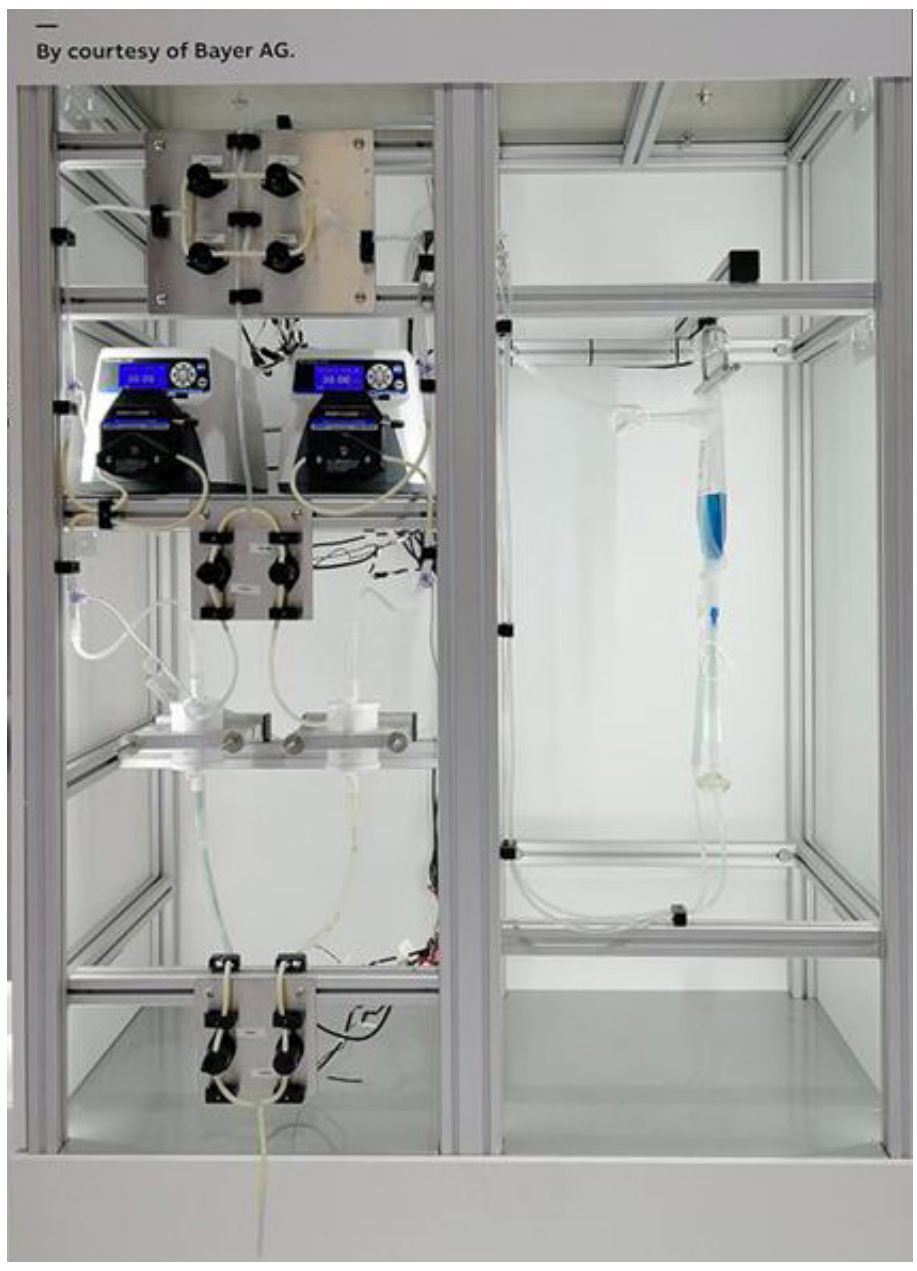

BILD 12: Prozessmodul zur Aufreinigung von Antikörpern
Spezialchemikalien machen. Im Fokus stehen dabei jene Themen, die aktuell die höchsten Entwicklungsanforderungen haben und für die Implementierung der Smart Factory unerlässlich sind, wie zum Beispiel die Zulassung und Automatisierung modularer, hochflexibler Systeme. Derzeit werden Pilotprojekte mit industriellen Partnern zum Thema Automatisierung modularer Systeme durchgeführt. In Zusammenarbeit mit Huber Kältetechnik wurde das Katalogmodul Temperature Control mit dem MTP in die Prozesssteuerungsebene für die Modulautomation integriert. Neben der Modulautomation von Spezialsystemen werden die Orchestrierung und der Betrieb der modularen Anlagen in der Prozessleitebene mit den Herstellern B\&R Industrial Automation und Siemens pilotiert.

Für die Modulautomation wurden in den B\&RModulen X20-Steuerungen und in den mit Siemens automatisierten Modulen Steuerungen aus der Familie Simatic S7-410 mit Simatic PCS 7 sowie kompakte mit TIA Portal projektierte Steuerungen aus der Familie S7-1500 eingesetzt.

In den gemeinsam durchgeführten Pilotprojekten wurde in der Praxis nachgewiesen, dass modulare Anlagen mittels der Konzepte der VDI/VDE/Namur 2658 technisch realisierbar sind. Die Erfahrungen zeigen, dass speziell dem Modulengineering eine hohe Bedeutung zukommt. Um in verschiedenen Anlagenszenarien einsetzbar zu sein, sind Dienste mit vielen Fahrweisen nötig. Dies hat einen vergleichbar hohen Aufwand im Modulengineering zur Folge. Abhilfe könnten Konzepte für ein Modul-DiensteUpgrade schaffen, mit welchen es möglich wäre, einen Dienst bei Bedarf um eine weitere Fahrweise zu ergänzen. Dies erfordert ein Versionsmanagement, welches in der VDI/VDE/Namur 2658 bisher nicht vorgesehen ist. Für Module kleinerer bis mittlerer Komplexität sowie Module mit mittlerer bis hoher Komplexität sind Steuerungen am Markt verfügbar. Auch praxistaugliche Konzepte zur Modul- und Dienste-Orchestrierung sind vorhanden. Ein Plug \& Configure von Modulen zu einer modularen Anlage ist mit den verfügbaren Leitsystemen von Siemens und B\&R heute realisierbar. Dies ist ein erster bedeutender Schritt zur Realisierung der Vision Plug \& Produce.

Neben den Aktivitäten im Rahmen der Pilotprojekte verantwortet Merck im Rahmen des Enpro2-ORCAProjekts das Arbeitspaket Genehmigung modularer Anlagen. Ziel des Arbeitspakets ist die Entwicklung eines Konzepts zum Behördenengineering modularer Anlagen. Dieses Konzept muss es unter Beachtung sicherheitstechnischer und regulatorischer Aspekte ermöglichen, dass die Genehmigung und Zertifizierung modularer Anlagen gegenüber heutigen Prozessen deutlich beschleunigt werden kann. Für die Demonstration sowie Evaluierung einzelner Arbeitsergebnisse 


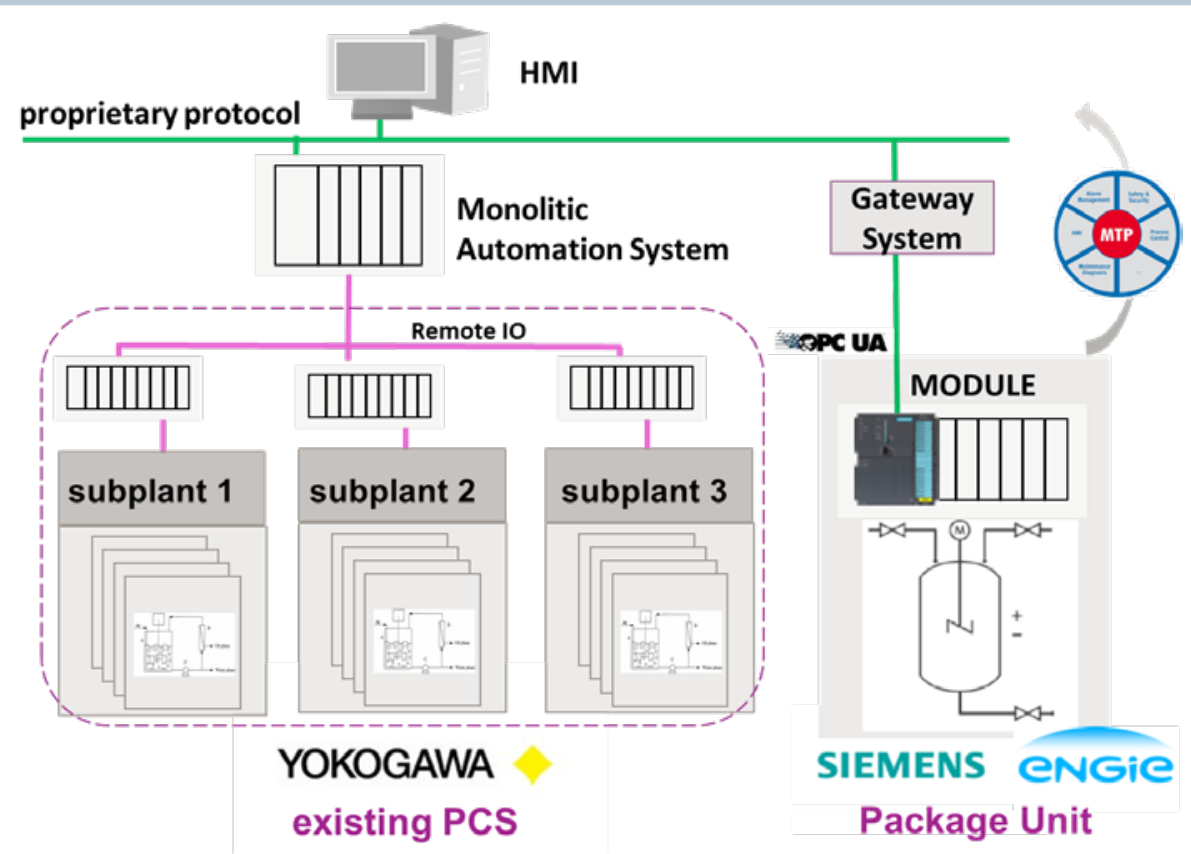

BILD 13: Package-Unit-Integration in ein bestehendes DCS mittels MTP

aus allen Arbeitspaketen werden im Rahmen des Projektes auch einige Demonstratoren erstellt.

\subsection{Achema-Demonstrator}

Die Verbände ZVEI, Namur, Dechema und VDMA haben gemeinsam mit akademischen und industriellen Partnern auf der Achema 2018 gezeigt, wie die flexible Produktion der Zukunft aussieht. Es wurden neueste Konzepte der Orchestrierung modularer Anlagen und innovative Visualisierungs- und Interaktionskonzepte vorgestellt. Durch die dargestellten Konzepte konnten die Vorteile der modularen Produktion aufgezeigt und mit fachkundigem Publikum diskutiert werden.

Zur Veranschaulichung der modularen Automation wurde ein aus drei intelligenten Process Equipment Assemblies (PEA) bestehender Demonstrator aufgebaut. In Bild 11 sind diese drei PEAs zu sehen, wie sie auf der Achema 2018 ausgestellt wurden. Von links startend sind ein Dosier-, Reaktor- und Temperiermodul aufgestellt. Die einzelnen intelligenten PEAs sind mit Controllern unterschiedlicher Hersteller (Wago, Siemens und Huber) ausgestattet, um die Interoperabilität zwischen den verschiedenen Herstellern zu demonstrieren.

Das Dosiermodul wurde vom Process-To-Order Lab der TU Dresden zur Verfügung gestellt. Die Hauptaufgabe im Anlagenverbund besteht im Dosieren einer Flüssigkeit in den Reaktor. Der Reaktor wurde von Merck entwickelt und durch eine austauschbare modulare Funktionseinheit (Functional Equipment Assembly, FEA) von Samson ergänzt. Dieses hierarchische Konzept dient dazu, auf unterschiedliche Stoffeigenschaften (zum Beispiel variante Viskosität) mittels einer austauschbaren FEA zu reagieren. Eine FEA ist für den POL nicht zu sehen, die Dienste werden von dem Reaktormodul angeboten. Das dritte Modul wurde von der Firma Huber Kältemaschinenbau bereitgestellt und ist ein Katalogmodul, das mit einem Embedded Controller ausgestattet ist. Auf diesem wurde das MTPKonzept implementiert. Das Huber-Modul ist für die Temperierung des Reaktors verantwortlich. Im Prozessmodul wurden mehrere Dienste umgesetzt, die das aktuelle Zustandsmodell verwenden, das im Vorfeld der Achema durch die Task Force Prozessführung spezifiziert wurde. Ziel war die Orchestrierung der modularen Anlage in einem diensteorientiertem Rezept.

Während der Planung, der Inbetriebnahme und der Orchestrierung wurden Erkenntnisse abgeleitet, die im Rahmen aktueller und zukünftiger Projekte (wie beispielsweise dem Enpro2-ORCA-Projekt [12]) aufgegriffen werden sowie in die Standardisierung des MTPs einfließen sollten. In [13] wurden die Aspekte der Kommunikation über OPC UA, der aktuellen Bedienund Visualisierungskonzepte sowie der Orchestrierung einer modularen Anlage ausgewertet. Während sich das im Rahmen der Task Force Prozessführung beschlossene Zustandsmodell als eine gute Grundlage erwiesen hat, benötigt die Kommunikation über OPC UA noch 
Engie

\section{Module Supplier Package Unit}

Cooling Machines

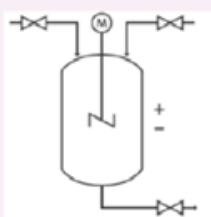

\section{Siemens}

\section{Module Automation Engineering}

PLC S7-1500

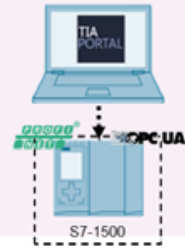

Yokogawa

\section{Plant Automation Engineering}

DCS Centum VP

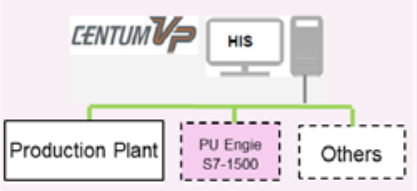

Evonik

\section{Plant Integrator End User}

BILD 14: Workflow der Integration von Package Units in ein DCS mittels MTP

weitere spezifische Definitionen und Absprachen. Ebenso führten die im Voraus definierten Betriebsmodi während der Inbetriebnahme zu Schwierigkeiten, die ein manuelles Eingreifen bis auf die IO-Ebene nötig machten.

Mit dem Achema-Demonstrator konnte gezeigt werden, dass sich der agile Ansatz aus Sprints und Demonstratoren für die Standardisierung des MTPs bewährt hat. Es konnten zahlreiche Lücken in der Spezifikation identifiziert und behoben werden, sodass ein praktisch erprobter Standard entsteht. Die festgestellten Forschungslücken werden im ORCAProjekt aufgegriffen und konzeptionell aufgearbeitet.

\subsection{Modulare Automation für biopharmazeutische Anwendungen}

In einem gemeinsamen Projekt haben Bayer, ABB, die TU Dresden und die HSU Hamburg eine Demonstrationsanlage errichtet, um die Realisierbarkeit modularer Automatisierung auf Basis der MTP-Idee zu demonstrieren. Die Anlage basiert auf einer modularen Pilotanlage zur kontinuierlichen Verarbeitung von biopharmazeutischen Produkten. Ziel des ursprünglichen Projekts ist es, durch die Austauschbarkeit von Prozessmodulen eine hohe Prozessflexibilität zu erreichen. Die Automatisierung war bisher klassisch umgesetzt worden. Somit war die prozessseitige Flexibilität in der Automatisierung nur bedingt abbildbar.

Im Verbundprojekt wurden die einzelnen Module mit eigenen Controllern ausgestattet. Die ursprüngliche Software wurde auf Modulebene heruntergebrochen und mit eigenen Bedienbildschirmen und Diensten ausgestattet. Die Prozessleitebene wurde durch das Prozessleitsystem 800xA realisiert, und die aus der Modulsoftware exportierten MTPs wurden in dieses System integriert. Dies ermöglicht die Visualisierung und den Betrieb der Module innerhalb des Prozessleitsystems. Durch die Implementierung von Diensten innerhalb der Module und deren Bekanntmachung durch das MTP im Prozessleitsystem wurde die dienstbasierte Steuerung des Prozesses ermöglicht.

\subsection{Nahtlose Package-Unit-Integration für} Brown- und Greenfield-Anwendungen

Evonik, Engie, Siemens und Yokogawa arbeiten derzeit an der Integration einer Kühlaggregat-Einheit in eine konventionelle Produktionsanlage mittels MTP. Dieses bietet eine Standardbeschreibung der Prozessmodule, um eine effiziente Integration in die Prozesssteuerungsebene zu gewährleisten. Das Konzept wird als Wegbereiter für die modulare Produktion betrachtet. Package Units von nicht modularen Anlagen weisen eine ähnliche Komplexität in Bezug auf die Integration auf wie Prozessmodule modularer Anlagen. Daher ist derselbe Mechanismus nicht nur für die modulare Produktion nützlich, sondern auch für die schnelle und sichere Integration von Package Units in herkömmliche Produktionsanlagen.

In diesem Pilotprojekt wurde das auf einer Steuerung der Familie Simatic S7-1500 basierende Automatisierungssystem (Siemens) der Kühlaggregat-Einheit (Engie) angepasst, um eine MTP-Exportdatei mit HMIund Kommunikationsaspekten zu erstellen. Diese MTP-Datei wurde in das Prozessleitsystem (Yokogawa) importiert. Somit konnten die Bedienbilder und die Kommunikationsschnittstelle automatisch generiert werden. 
Folgende Systeme wurden im Projekt verwendet:

- Siemens (SPS-Lieferant für Package Unit): Steuerung S7-1500 mit integriertem OPC-UA-Server; TIA Portal V15 mit MTP-Exporttool gemäß VDI/VDE/ Namur 2658,

n Yokogawa (DCS-Lieferant): Entwicklung eines MTPImporttools gemäß VDI 2658; Unified Gateway System mit OPC-UA-Kommunikation,

- Engie (Package Unit Supplier): Ergänzung des vorhandenen SPS-Programms um von Siemens bereitgestellte MTP-Koppelbausteine zur Realisierung der MTP-Schnittstelle.

Die Inbetriebnahme am Evonik-Produktionsstandort wird voraussichtlich im ersten Quartal 2019 beginnen. Mit diesem Projekt wird erwartet, dass das MTP-Informationsmodell für die Integration von Package Units angewendet werden kann. Dadurch ist eine Reduzierung des manuellen Aufwands und damit eine Einsparung von Zeit und Kosten vorgesehen.

\section{ZUSAMMENFASSUNG UND AUSBLICK}

Seit 2015 arbeiten die unterschiedlichen Arbeitskreise an der Spezifizierung des MTPs, einer funktionalen Beschreibung von eigenständig automatisierten Prozesseinheiten zur flexiblen Integration in die Prozessorchestrierungsebene (POL). Durch die Überführung der Spezifikationen in die Richtlinie VDI/VDE/Namur 2658 konnte ein wichtiger Meilenstein erreicht werden. Auch wenn der bisherige Stand der Richtlinie nur einige grundlegende Aspekte des Modul- und Anlagenengineerings umfasst, haben

\section{REFERENZEN}

[1] Zentralverband Elektrotechnik- und Elektronikindustrie e. V., ZVEI (2015). Module-Based Production in the Process Industry - Effects on Automation in the "Industrie 4.0" Environment: Recommendations of the Modular Automation Working Group Following Namur Recommendation NE 148. Abgerufen von: https://www. zvei.org/fileadmin/user_upload/Presse_und_Medien/ Publikationen/2015/maerz/White_Paper_-ModuleBased_Production_in_the_Process_Industry_-Effects_on_Automation_in_the__Industrie_4.0_Environment/

Module-Based_Production_in_the_Process_Industrywhitepaper-zvei.pdf

[2] Bernshausen, J., Haller, A., Holm, T., Hoernicke, M., Obst, M., \& Ladiges, J. (2016). Namur Modul Type Package - Definition. atp edition, 58(01-02), 72-81. doi:10.17560/atp.v58i01-02.554

[3] Obst, M., Holm, T., Urbas, L., Fay, A., Kreft, S., Hempen, U., \& Albers, T. (2017). Beschreibung von Prozessmodulen. atp edition, 57(01-02), 48-59. doi:10.17560/atp.v57i01-02.473

[4] NAMUR. (2013). NE 148: Anforderungen an die Automatisierungstechnik durch die Modularisierung verfahrenstechnischer Anlagen. Namur: www.namur.net

[5] Bloch, H., Hensel, S., Hoernicke, M., Stark, K., Menschner, A., Urbas, L., Fay, A., Knohl, T., Bernshausen, J., \& Haller, A. (2017). Zustandsbasierte Prozessführung modularer Prozessanlagen. atp edition, 59(10), 46-57. doi:10.17560/atp.v59i10.1899
[6] IEC 61131-3. (2013). Grundlagen Speicherprogrammierbarer Steuerungen, Teil 3: Programmiersprachen. IEC: www.iec.ch

[7] VDI/VDE/NAMUR 2658 Blatt 1. (2017). Automatisierungstechnisches Engineering modularer Anlagen in der Prozessindustrie, Blatt 1: Allgemeines Konzept und Schnittstellen. VDI: www.beuth.de

[8] VDI/VDE/NAMUR 2658 Blatt 2. (2018). Automatisierungstechnisches Engineering modularer Anlagen in der Prozessindustrie, Blatt 2: Modellierung von Bedienbildern. VDI: www.beuth.de

[9] VDI/VDE/NAMUR 2658 Blatt 3. (o.J.). Automatisierungstechnisches Engineering modularer Anlagen in der Prozessindustrie, Blatt 3: Bibliothek für Datenobjekte, Entwurf. VDI: www.beuth.de

[10] VDI/VDE/NAMUR 2658 Blatt 4. (o.J.). Automatisierungstechnisches Engineering modularer Anlagen in der Prozessindustrie, Blatt 4: Modellierung von Moduldiensten (Entwurf). VDI: www.beuth.de

[11] NAMUR. (2017). NE 107: Selbstüberwachung und Diagnose von Feldgeräten. Namur: www.namur.net

[12] Menschner, A., Hensel, S., da Silva Santos, P., Schäfer, C., Stark, K., Scheffler, S., ... \& Urbas, L. (2018). Lessons Learned aus dem Achema-Demonstrator. Jahrestreffen der ProcessNet-Fachgemeinschaft "Prozess-, Apparateund Anlagentechnik" (PAAT). 12.-13.11.2018, Köln.

[13] DECHEMA Gesellschaft für Chemische Technik und Biotechnologie e. V. (2018). Effiziente Orchestrierung modularer Anlagen. Abgerufen von: http://enproinitiative.de/ORCA.html 


\section{AUTOREN}

Dr.-Ing. JENS BERNSHAUSEN (geb. 1980) ist Lead Engineer in der Cooperate Function Engineering \& Technology der Bayer AG. Seit 2013 befasst er sich mit dem Engineering von modularen Produktionsanlagen im chemischen und pharmazeutischen Umfeld. Er ist Obmann des Namur-Arbeitskreises 1.12 „Module / Plug \& Produce“.

Bayer AG

Engineering \& Technology, Building K 9,

51368 Leverkusen,

Tel: +49 (0) 2143072697 ,

E-Mail: jens.bernshausen@bayer.com

Dipl.-Ing. (FH) AXEL HALLER (geb. 1961) ist Protfolio- und Produktmanager im Geschäftsbereich Chemie Öl und Gas von ABB. Er verfügt über langjährige Erfahrung im Bereich Automatisierung von Prozessanlagen, sowohl im Consulting, wie auch im Vertrieb und der Abwicklung.

M.SC. HENRY BLOCH (geb. 1988) arbeitet seit 2015 als wissenschaftlicher Mitarbeiter am Institut für Automatisierungstechnik an der Helmut-SchmidtUniversität/Universität der Bundeswehr Hamburg. Sein Forschungsschwerpunkt liegt in der Entwicklung von Steuerungskonzepten modularer Anlagen. Ab April 2019 gründet er gemeinsam mit Anna Menschner und Stephan Hensel das Startup Semodia, das Software zu MTP-Generierung und -Editierung sowie MTPs für die Labor- und Technikumstechnik anbietet.

Dipl.-Ing. (FH) MARIO HOERNICKE (geb. 1984) ist Principal Scientist am ABB Forschungszentrum in Ladenburg. Sein Forschungsschwerpunkt umfasst die Entwicklung neuer und innovativer Konzepte im Bereich digitaler Anlagen und modularer Automatisierungstechnik.

Dipl.-Ing. STEPHAN HENSEL (geb. 1990) arbeitet seit 2015 als wissenschaftlicher Mitarbeiter am Lehrstuhl für Prozessleittechnik/AG Systemverfahrenstechnik der Technischen Universität Dresden. Sein Forschungsgebiet umfasst den Umgang mit Anforderungsänderungen in industriellen Informationsmodellen. Ab April 2019 gründet er gemeinsam mit Anna Menschner und Henry Bloch das Startup Semodia, das Software zu MTP-Generierung und -Editierung sowie MTPs für die Labor- und Technikumstechnik anbietet.

Dipl.-Ing. ANNA MENSCHNER (geb. 1987) arbeitet seit 2014 als wissenschaftliche Mitarbeiterin an der Professur für Prozessleittechnik/AG Systemverfahrenstechnik der Technischen Universität Dresden. Ihre Forschungsschwerpunkte liegen in der Untersuchung von Virtuell Funktionalen Modulen für die Prozessindustrie. Ab April 2019 gründet sie gemeinsam mit Henry Bloch und Stephan Hensel das Startup Semodia, das Software zu MTP-Generierung und -Editierung sowie MTPs für die Labor- und Technikumstechnik anbietet.

Dipl.-Ing. ANDREAS STUTZ (geb. 1990) arbeitet als Forschungsingenieur in der Vorfeldentwicklung bei Siemens in Karlsruhe mit dem Schwerpunktthema zukünftige Leitsystemarchitekturen. Nach seiner Berufsausbildung als Automatisierungstechniker bei Siemens, absolvierte er den Bachelor-Studiengang in Energie- und Automatisierungstechnik und schloss 2015 den Master-Studiengang Elektrotechnik mit dem Schwerpunkt Automatisierungstechnik ab.

Dr.-Ing. MATHIAS MAURMAIER (geb. 1978) betreut als Projektleiter die Themen Geräteintegration sowie offene und modulare Automatisierungsarchitekturen in der Vorfeldentwicklung bei Siemens in Karlsruhe. Nach dem Studium der Elektrotechnik promovierte er im Themenfeld Modellgetriebene Entwicklung von Automatisierungssystemen am Institut für Automatisierungs- und Softwaretechnik der Universität Stuttgart.

Dr.-Ing. THOMAS HOLM (geb. 1979) leitet den Bereich Innovation \& Technology bei WAGO Kontakttechnik in Minden. Ein thematischer Schwerpunkt liegt unter anderem in der effizienten Automatisierung wandelbarer Produktionssysteme.

Dipl.-Ing. (FH) CHRISTIAN SCHÄFER (geb. 1982) ist Senior Engineer in der Integrated Supply Chain der Sparte Performance Materials der Merck KGaA in Darmstadt. Seit 2008 befasst er sich im Rahmen der Verfahrensentwicklung mit dem Engineering und Betrieb von modularen Anlagen im chemischen Umfeld. Er ist Mitarbeiter in dem Namur-Arbeitskreises 2.3 „Batch Control“.

Prof. Dr.-Ing. LEON URBAS (geb. 1965) leitet an der TU Dresden die Professur für Prozessleittechnik und die Arbeitsgruppe Systemverfahrenstechnik und ist Sprecher des GMA-FA 5.16. Forschungsfelder sind offene Informations- und Automationsarchitekturen und Mensch-Technik-Kollaboration in der Prozessindustrie. 


\section{AUTOREN}

Dr.-Ing. ULRICH CHRISTMANN (geb. 1966) verfügt über langjährige Erfahrung im Bereich Automatisierungstechnik in unterschiedlichen BetreiberFunktionen mit wachsender Verantwortung. Derzeit ist er als Key Expert in der Corporate Function Engineering \& Technology der Bayer AG für die kontinuierliche Weiterentwicklung der Automation Strategy verantwortlich. In der Namur ist er als Leiter des Arbeitsfeldes 1 „Project Planning and Construction“ verantwortlich für die Fortentwicklung des Engineering-Prozesses.

Dr.-Ing. CHRISTOPH FLEISCHER-TREBES (geb. 1986) arbeitet seit 2016 als Project Engineer bei der INVITE GmbH und ist für die konzeptionelle Pla- nung und das Engineering von modularen Anlagen zuständig. In seiner Promotion hat er sich mit den Themen Planung, Sicherheit und Zuverlässigkeit von modularen Anlagen befasst.

Dr.-Ing. FRANK STENGER (geb. 1972) ist Leiter der Gruppe „Chemical Reaction \& Polymer Technology Optimization“ bei der Evonik Technology \& Infrastructure $\mathrm{GmbH}$. Er ist verantwortlich für die Entwicklung modularer Produktionsanlagen. Innerhalb der ProcessNet/Dechema leitet er den temporären Arbeitskreis „Modular Plants“. Zudem ist er Stellvertretender Vorsitzender der Fachgruppe „Micro-Process Technology“. unterschiedliche Pilotprojekte gezeigt, dass das MTPbasierte Engineering von Produktionsanlagen in der Praxis funktioniert - auch wenn einige Aspekte der Integration aufgrund fehlender Spezifikationen noch herkömmlich erfolgen müssen. Vorteile bei der Implementierung hinsichtlich Engineeringeffizienz und Kostenersparnis sowie eine wesentliche Reduzierung der Anlagenumbauzeiten konnten bestätigt werden.

Basierend auf der Richtlinie VDI/VDE/Namur 2658 haben einige Automatisierungslieferanten für 2019 kommerziell erhältliche Engineeringwerkzeuge angekündigt, die ein MTP-basiertes Engineering auf Modulund Anlagenebene ermöglichen.
Für die Erprobung des MTPs ist es notwendig, die Funktionen in der Praxis umzusetzen und Erfahrungen zu sammeln. Mit der Kommerzialisierung der Produkte ist es nun möglich, die MTP-Funktionalitäten zur Integration von Package Units und Prozessmodulen im Projektalltag anzufragen und zu verwenden. An dieser Stelle sind die Anwender gefragt, auf die Automatisierungslieferanten zuzugehen und gemeinsam den neuen Weg der flexiblen Anlagenautomatisierung einzuschlagen.

Ein Teil der in diesem Artikel vorgestellten Arbeiten wird im Rahmen der ENPRO 2.0 Initiative vom BMWi finanziell unterstützt (FKZ 03ET1517A). 